\title{
Downregulation of the $\mathrm{N}$-myc downstream regulated gene 1 is related to enhanced proliferation, invasion and migration of pancreatic cancer
}

\author{
GANG CEN ${ }^{*}$, KUNDONG ZHANG ${ }^{*}$, JUN CAO and ZHENGJUN QIU \\ Department of General Surgery, Shanghai General Hospital of Nanjing Medical University, \\ 100 Haining Road, Shanghai 200080, P.R. China
}

Received August 10,2016; Accepted December 27, 2016

DOI: $10.3892 /$ or.2017.5355

\begin{abstract}
The N-myc downstream regulated gene 1 (NDRG1) is differently expressed in human malignancies according to the tumor type. We investigated the expression of NDRG1 in pancreatic cancer tissues and cell lines as well as how it affects tumor growth, invasion and migration in pancreatic cancer cells. Experimental groups included NDRG1 overexpression and knockdown pancreatic cancer cell lines. Lentivirus-based empty vector transfected cells (NC group) were considered control groups. Proliferation, invasion and migration related proteins such as STAT3, MMPs, PTEN, PI3K/AKT were assessed by CCK-8, Transwell assay and western blotting. Efficient NDRG1 overexpression results in reduced cell proliferation, invasion and migration. Inversely, downregulation of NDRG1 promoted proliferation, invasion and migration. We also found NDRG1 could deactivate p-STAT3, PI3K, p-AKT, MMP2, MMP9 and activate PTEN. NDRG1 is a potential anti-oncogene. Its upregulation significantly decreases pancreatic cancer tumorigenesis, likely by inhibiting STAT3 and the PI3K/AKT signaling pathway.
\end{abstract}

\section{Introduction}

Pancreatic cancer is the most intractable disease among human cancers, with a low 5-year survival rate ranging from 5 to $6 \%(1,2)$. Pancreatic cancer patients are asymptomatic so that nearly $85 \%$ cases are diagnosed at a late stage accompanied by blood vessel invasion or distant metastasis, thus are not available for surgical resection (3). Even for the few

Correspondence to: Dr Jun Cao or Dr Zhengjun Qiu, Department of General Surgery, Shanghai General Hospital, 100 Haining Road, Shanghai 200080, P.R. China

E-mail: caojdoctor@sina.com

E-mail: qiuzjdoctor@sina.com

*Contributed equally

Key words: NDRG1, pancreatic cancer, STAT3, PTEN patients who underwent a potentially curative resection, the long-term survival remains disappointing due to early recurrence or metastatic disease (4). Hence, a better understanding of the molecular mechanisms underlying pancreatic cancer progression is needed to investigate and design more effective therapies.

$\mathrm{N}$-myc downstream-regulated gene 1 (NDRG1) is a member of the $\mathrm{N}$-myc downregulated gene family which belongs to the $\alpha / \beta$ hydrolase superfamily. Previous studies found that NDRG1 was downregulated in colorectal (5), gastric (6), cervical (7), ovarian (8) and prostate (9) cancer. Moreover, it has been reported that NDRG1 played a tumor suppressive role in a variety of tumorigenic signaling pathways. For example, Chen et al found that NDRG1 inhibits the TGF- $\beta$-induced EMT by maintaining E-cadherin and $\beta$-catenin at the cell membrane that cause decreased vimentin and ability of cancer cell migration and invasion (10). NDRG1 inhibits phosphorylation and nuclear translocation of $\beta$-catenin which leads to increased cell-cell adhesion and inhibition of the WNT pathway (9). Overexpression of NDRG1 in cancer cells not only induces differentiation but also reduces invasion and metastasis (10). Several studies further reported that a positive correlation between NDRG1 expression and patient prognosis, indicating NDRG1 may be a prognostic biomarker in prostate (11) and colorectal cancer (12). Although NDRG1 was largely regarded as a metastasis suppressor, there are also some studies revealing that NDRG1 is positively associated with cancer cell proliferation, differentiation and metastasis. In hepatocellular carcinoma, NDRG1 is significantly associated with advanced tumor stages and poor survival of patients by promoting cancer cell growth and preventing $\beta$-catenin degradation (13). In lung cancer, NDRG1 is upregulated by HIF-1 $\alpha$ and its overexpression results in more proliferation and less apoptosis of cancer cells (14). These above results may implicate the tissue-specific effects of NDRG1, which suggests that this protein plays pleiotropic roles in cancer progression. While in pancreatic cancer, the biological functions of NDRG1 in the pathogenesis of pancreatic cancer has not been investigated in detail. In this study we explored the expression of NDRG1 in pancreatic cancer and elucidate the effects of NDRG1 on the invasion and migration capability of pancreatic cancer cell lines. In addition, we 
examined whether change of those tumor related capability could be influenced by NDRG1 mediated STAT3, MMP2, MMP9 expression.

\section{Materials and methods}

Cell lines and culture. The human pancreatic cancer cells, including MIA-PACA-2, ASPC-1, BXPC-3, CAPAN-1, CAPAN-2, CFPAC-1, HPAF-II, HS766T, PANC1 and SW1990 were obtained from the Americacn Type culture Collection (ATCC, Manassas, VA, USA). PANC1, SW1990, CAPAN-2, HS766 T and MIA-PACA-2 cell lines were maintained in DMEM with 10\% FBS (Gibco, Carlsbad, CA, USA). ASPC-1, BXPC-3, CFPAC-1, HPAF-II cell lines were maintained in 1640 with $10 \%$ FBS. CAPAN-1 cell line was maintained in IMDM with $10 \%$ FBS. The cells were incubated in a humidified atmosphere of $5 \% \mathrm{CO}_{2}$ and $95 \%$ air.

$R N A$ extraction and $q R T-P C R$. Total RNA was extracted from pancreatic tissues, adjacent normal tissues and cell lines using TRIzol reagent (Invitrogen, USA) according to the manufacturer's instructions. cDNA was synthesized using the Prime-Script RT reagent kit (Tiangen, Beijing, China). qRT-PCR was performed using SYBR Green PCR Master Mix (Takara, Dalian, China) on an ABI 7500 fast real-time PCR system (Applied Biosystems, Foster City, CA, USA). Expression data were uniformly normalized to the internal control U6 and the relative expression levels were evaluated using the $\Delta \Delta \mathrm{Ct}$ method. The primers for NDRG1 were 5'-CTC TGTTCACGTCACGCTGT-3' (forward) and 5'-CTCCACCA TCTCAGGGTTGT-3' (reverse) and for GAPDH were 5'-GGA CCTGACCTGCCGTCTAG-3' (forward) and 5'-GTAGCCCA GGATGCCCTTGA-3' (reverse) according to the human NDRG1 and GAPDH cDNA sequences in GenBank. The GAPDH mRNA level was used for normalization.

Western blotting. Cell lysates were prepared by SDS lysis solution. Protein concentration was measured using a BCA protein assay kit. Equal amount of protein was separated by electrophoresis on a $10 \%$ SDS-polyacrylamide gel. The proteins were electrotransferred from the gel to nitrocellulose membrane. The membrane was blocked with $5 \%$ non-fat milk solution for $1 \mathrm{~h}$, and then was incubated with primary monoclonal antibody against NDRG1 (Abcam), $\beta$-actin (Calbiochem) at $4^{\circ} \mathrm{C}$ overnight. $\beta$-actin was used as an internal control. After washing with TBS-T, the membrane was incubated with secondary antibodies against rabbit immunoglobulin G. The membrane was washed and detected by the enhanced chemiluminescence (ECL) detection system (Thermo Fisher Scientific) according to the manufacturer's instructions.

Construction of the NDRG1 expression vector pcDNA3.1(+)/ NDRG1 and shRNA. The primer sequences used for NDRG1 expression were: NDRG1 forward, 5'-TTAGATCATGTCTCG GGAGATGCAGGAT-3'; and reverse 5'-TTGAATTCCTAGC AGGAGACCTCCATGG-3. The PCR product was then cloned into pcDNA3.1(+) (Invitrogen) using standard techniques. Either the NDRG1 expression vector pcDNA3.1(+)/NDRG1 or empty vector pcDNA3.1(+) was transiently transfected into
MIA-PACA2 and PANC1 cells using Lipofectamine 2000 (Invitrogen) according to the manufacturer's instructions. For the cell proliferation assay, cells were seeded into a 96-well plate $\left(2 \times 10^{4}\right.$ cells/well), and positive colonies were selected with G418 (Invitrogen) supplemented with growth medium. NDRG1 short hairpin RNA (shRNA) target sequence was: 5'-GCATTATTGGCATGGGAAC-3'. Double-stranded DNA coding NDRG1 shRNA was cloned into pRNATH1.1 Adeno shuttle vector containing a cGFP marker (Genscript). Adenovirus was packaged in Ad-293 cells (Agilent) and purified by $\mathrm{CsCl}_{2}$ gradient ultracentrifugation. Viral particle titer was determined by plague assay. For adenoviral transduction, MIA-PACA-2 and PANC1 cell was transduced with 100 multiplicity of infection of adenoviral control or shRNA for 24-48 h. Stable clones were confirmed by real-time RT-PCR and western blotting.

Wound healing assay. Cells from each group (NDRG1 overexpression and control, NDRG1 shRNA and control) were seeded into 6 -well plates $\left(5 \times 10^{5}\right.$ cells/well). The confluent monolayer was starved overnight, and then a single, linear scratch was created using a $20-\mu 1$ pipette tip. After wounding, the cells were washed gently with PBS to remove cell debris and placed in fresh DMEM supplemented with 0.1\% FBS to block cell proliferation. Images were captured using a phase contrast microscope at 0 and $48 \mathrm{~h}$. Wound closure was expressed as a percentage of the wound area at $0 \mathrm{~h}$.

Cell migration and invasion assays. Invasion assays were performed using the BD BioCoat ${ }^{\mathrm{TM}}$ Matrigel chamber in 24-well plates (BD, USA). Resuspension solution (100 $\mu \mathrm{l})$ containing $1 \times 10^{5}$ cells in DMEM with $1 \%$ FBS was added to the upper chamber, and $600 \mu \mathrm{l}$ DMEM supplemented with $10 \%$ FBS was added to the bottom chamber. After a 48-h incubation at $37^{\circ} \mathrm{C}$ in a $5 \% \mathrm{CO}_{2}$ incubator, cells in the upper well were wiped off using a cotton swab. Cells in the lower chamber were fixed, stained with H\&E, and counted under a light microscope. The migration assay was performed in a similar manner except that the chambers were covered without Matrigel.

Cell proliferation assay. Cell proliferation assay was performed with Cell Counting Kit-8 (Dojindo, Kumamoto, Japan) according to the manufacturer's instructions. Briefly, indicated pancreatic cancer cells were seeded in 96-well plates (1 $10^{4}$ cells/well) $24 \mathrm{~h}$ post-transfection and cultured in the growth medium. Cells were examined at $0,24,48,72,96$ and $120 \mathrm{~h}$. CCK-8 $(10 \mu \mathrm{l})$ was added to each well at different timepoints. After an incubation of $2 \mathrm{~h}$ at $37^{\circ} \mathrm{C}$, absorbance was measured at $450 \mathrm{~nm}$.

Statistical analysis. Statistical analysis was performed using GraphPad Prism version 4.02 software (GraphPad Software Inc., La Jolla, CA, USA) or SPSS 13.0 software (SPSS, Inc., Chicago, IL, USA). Values are expressed as the mean \pm standard deviation. Comparisons between multiple groups were made using a one-way analysis of variance, followed by t-test. $\mathrm{P}<0.05$ was considered to indicate a statistically significant difference between values. All experiments were conducted at least in triplicate. 
A

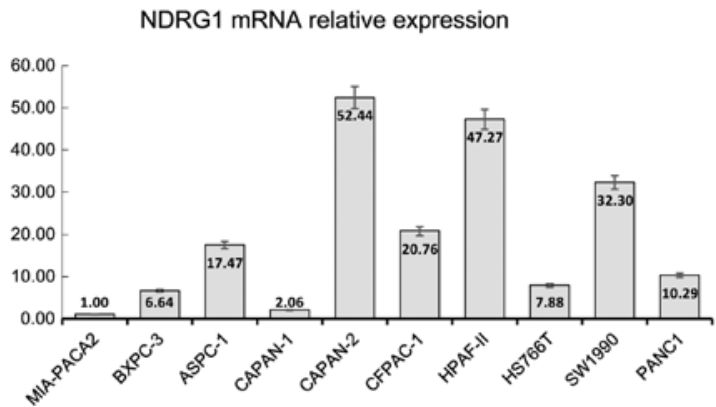

B

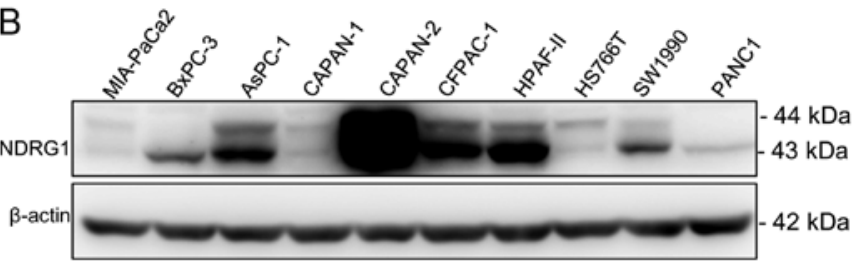

Figure 1. The NDRG1 expression profiles in 10 pancreatic cancer cell lines (A) qRT-PCR detection of NDRG1 mRNA levels. (B) Western blotting of NDRG1 protein.

\section{Results}

Cell models for NDRG1 cellular functions. In order to select suitable cell lines for subsequent upregulation or downregulation of NDRG1 and to observe these effects on proliferation, invasion, migration of cancer cells, we assessed NDRG1 expression in pancreatic cancer cell lines. As shown in Fig. 1, the expression of NDRG1 mRNA and protein were slightly mismatched. The top three highest mRNA expressions were CAPAN-2, HPAF-II, SW1990 but in NDRG1 protein expression, CAPAN-2, HPAF-II, PANC1 is the highest. Whereas, the lowest expression of mRNA and protein were MIA-PaCa2, BxPC-3, CAPAN-1 and MIA-PaCa2, CAPAN-1, HS766T, respectively (Fig. 1).

We employed PANC-1 and MIA-PaCa2 transfected with NDRG1 expressing plasmid and shRNA as cell models for the following in vitro study. After transfection with $48 \mathrm{~h}$, cells were lysed and subjected to qRT-PCR and western blotting for detecting NDRG1 expression. As indicated in Fig. 2, NDRG1 mRNA and protein were significantly elevated after transfection with NDRG1 expressing plasmid in PANC-1 and MIA-PaCa2 cell lines in comparison with the vector control cells, while the levels of NDRG1 were reduced remarkably with NDRG1 shRNA transfection as compared with controls. These data suggest that overexpression and knockdown of DRG1 in pancreatic cancer cell lines were established and ready for in vitro experiments.

NDRG1 inhibits cell proliferation, migration and invasion in pancreatic cancer cell lines. Wound scratch assays and Transwell assays were performed to evaluate the influence of NDRG1 on pancreatic cancer migration and invasion. As
A NDRG1 mRNA relative expression

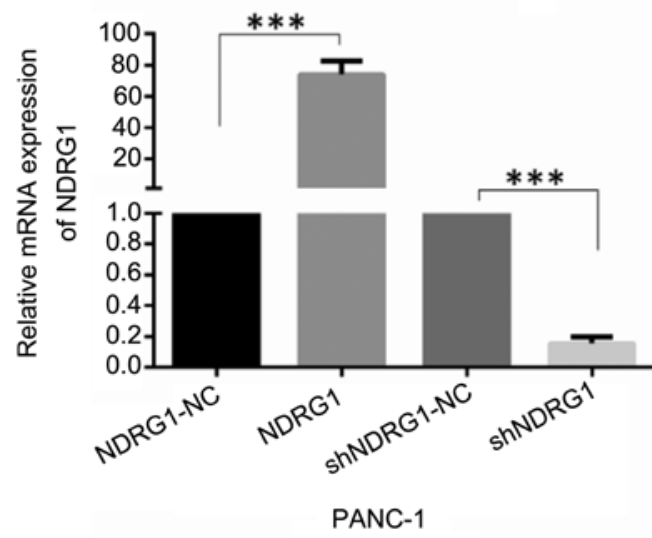

B

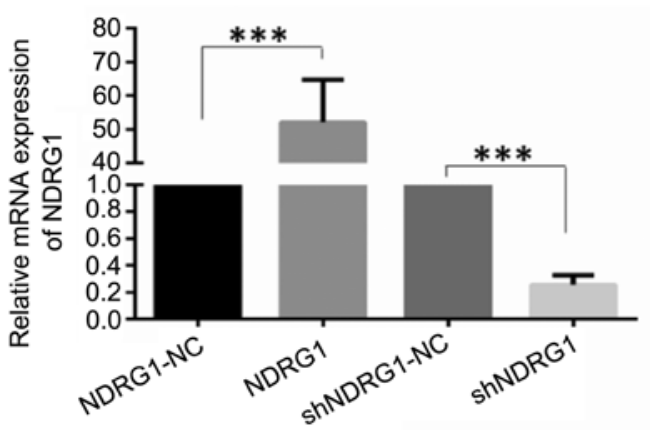

PANC-1
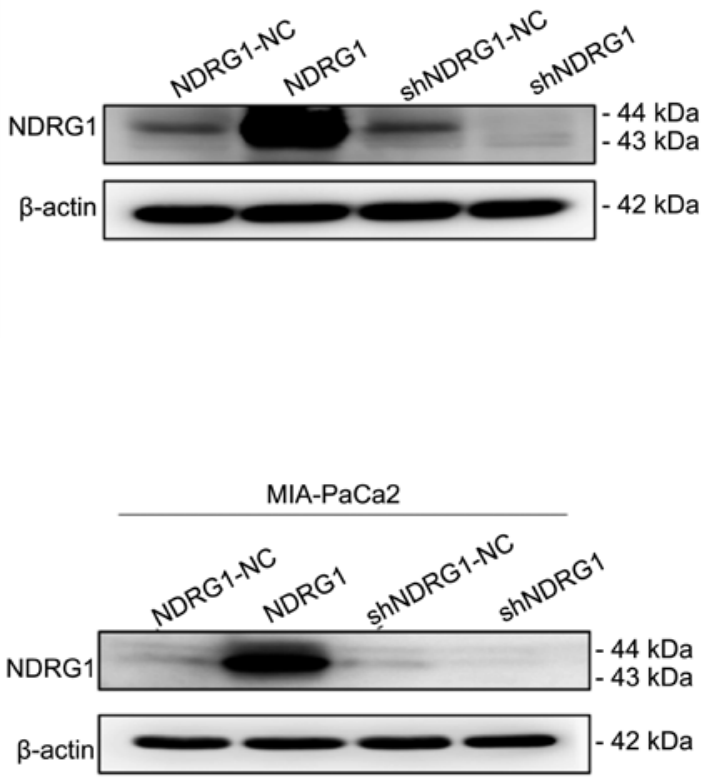

MIA-PaCa2

Figure 2. NDRG1 mRNA and protein expressions detection in PANC-1 (A) and MIA-PaCa-2 (B) cell lines after transfection with NDRG1 overexpression plasmid and shRNA. ${ }^{* * *} \mathrm{P}<0.001$. 
A Wound scratch assays
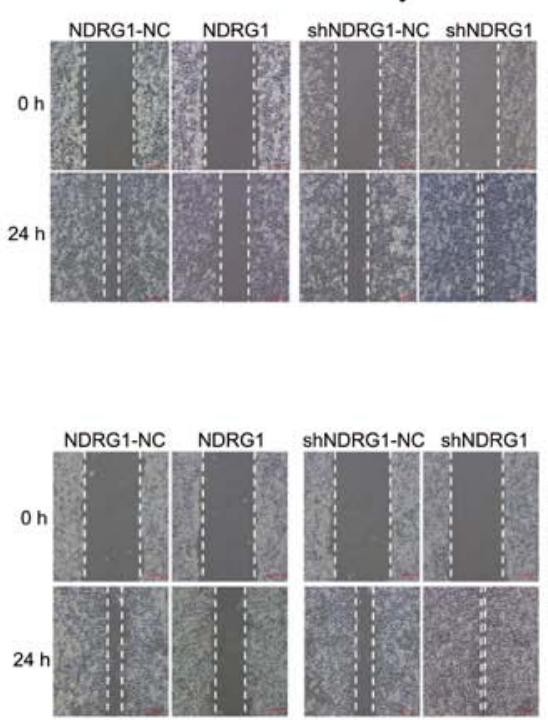

C Invasion assays

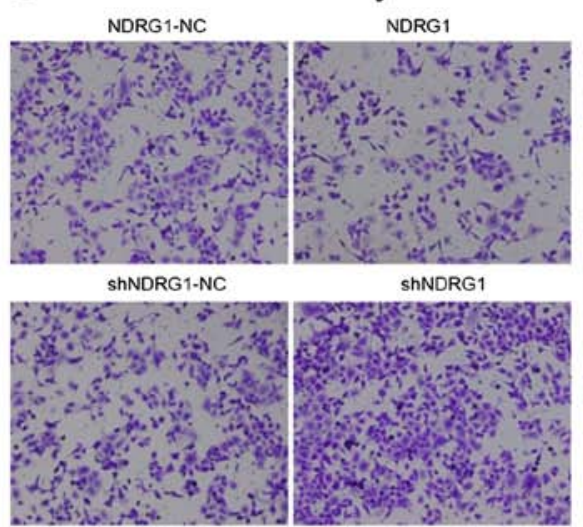

NDRG1-NC

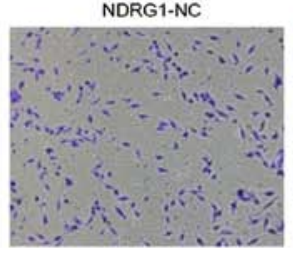

ShNDRG1-NC

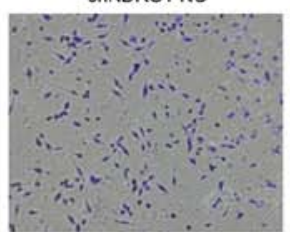

NDRG1

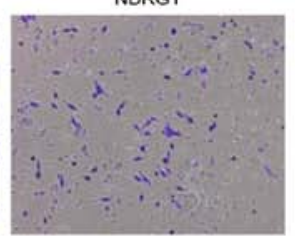

ShNDRG1

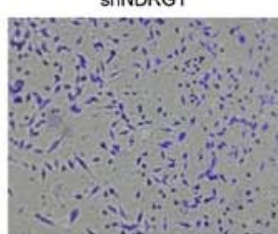

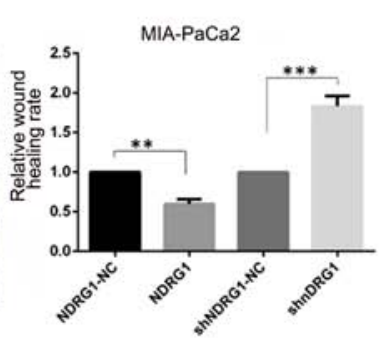

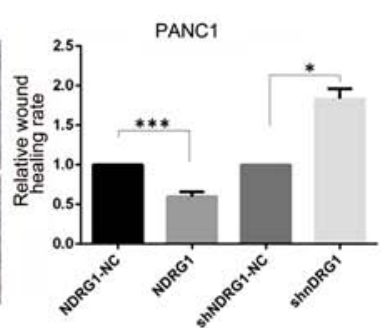

B Migration assays

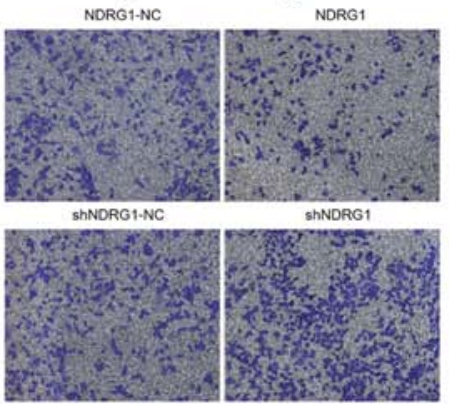

NDRG1

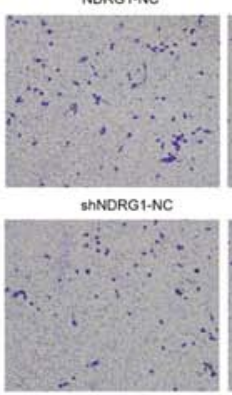

D
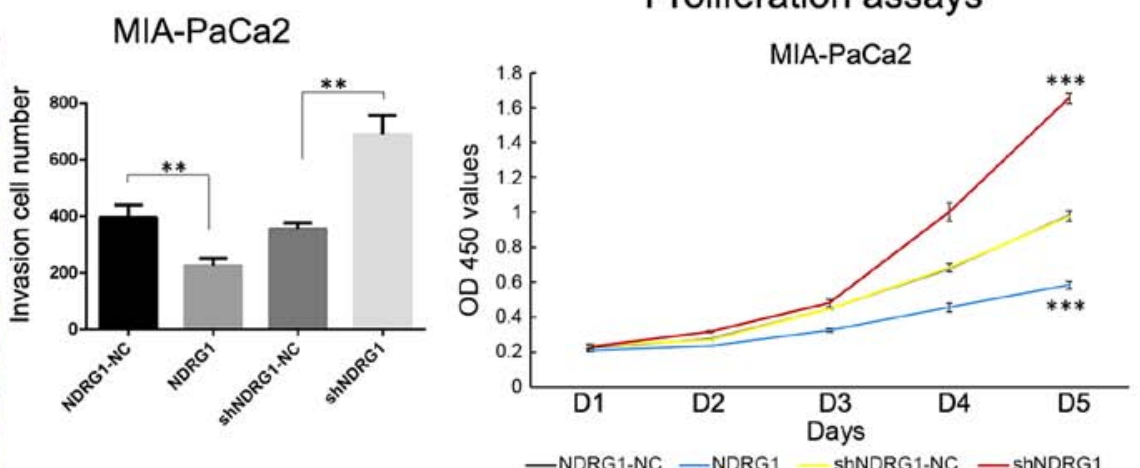

PANC1

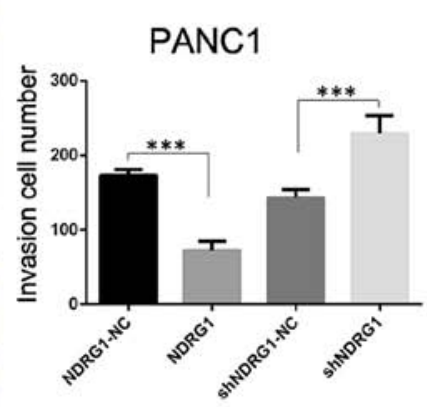

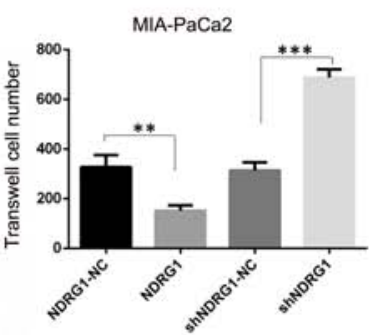

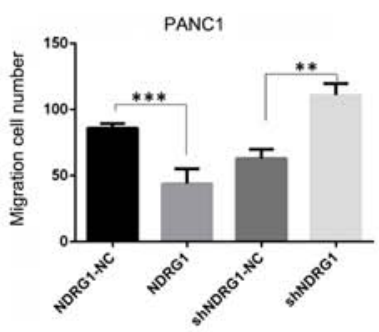

Figure 3. Effects of NDRG1 on pancreatic cancer MIA-PACA2 and PANC1 migration, invasion and proliferation. (A) Representative images of wound scratch assay at $24 \mathrm{~h}$ after transfection of NDRG1 overexpressing plasmid or NDRG1 shRNA and control. The percentage of wound healing distance is shown as compared with respective control cells. (B and C) Representative images of Transwell assays at $48 \mathrm{~h}$ after transfection of NDRG1 overexpressing plasmid or NDRG1 shRNA and control. The number of migrated or invaded cells is shown by comparing with respective control cells. (D) Effects of NDRG1 knockdown and overexpression on the proliferation of pancreatic cancer cells. Cell proliferation of untransfected cells and cells transfected with NDRG1 overexpressing plasmid or NDRG1 shRNA and control was assessed by CCK-8 assay. ${ }^{*} \mathrm{P}<0.05,{ }^{* *} \mathrm{P}<0.01,{ }^{* * * *} \mathrm{P}<0.001$.

shown in Fig. 3, the distance changes were calculated by defining the change of control cells as $100 \%$. Significant wound closure inhibition was observed in the NDRG1 overexpression
PANC-1 (1.00 vs $0.60 \pm 0.05, \mathrm{P}=0.0035)$ and MIA-PaCa2 $(1.00$ vs $0.58 \pm 0.055, \mathrm{P}=0.0001)$ cells. However, those with NDRG1 siRNA transfection migrated faster than control cells (PANC-1: 


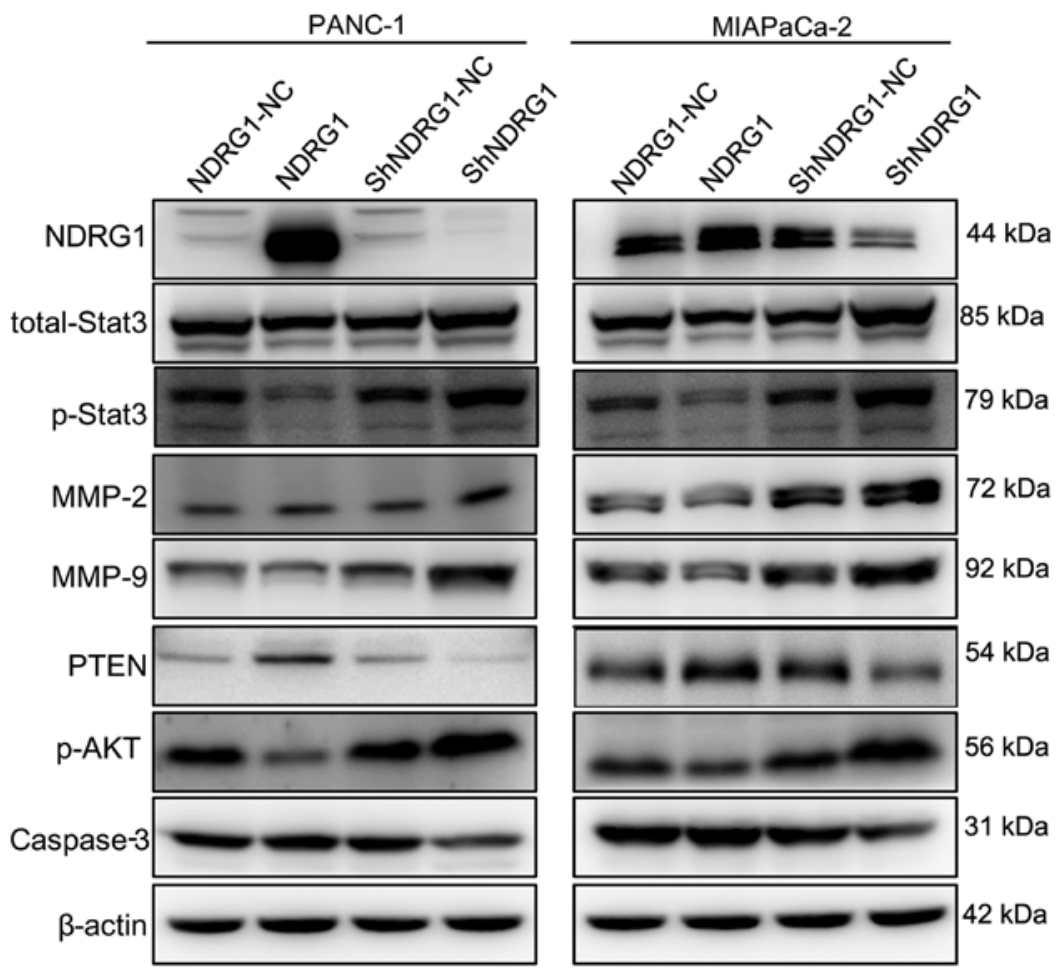

Figure 4. NDRG1 affects p-STAT3, MMP2, MMP9, PTEN, p-AKT and PI3K. Changes in expression levels of migration, invasion and proliferation related proteins following transfection with shNDRG1 or NDRG1 overexpressing plasmid in pancreatic cancer cells. $\beta$-actin was used as the internal control.

1.00 vs $1.83 \pm 0.1, \mathrm{P}=0.0003$; MIA-PaCa2: 1.00 vs $1.48 \pm 0.18$ $\mathrm{P}=0.01)$. Furthermore, Transwell assays with or without Matrigel were used to examine PANC-1 and MIA-PaCa2 cell properties of invasion and migration. In Transwell assays, the numbers of migrated and invaded PANC-1 (migration: $302.67 \pm 23.03$ vs $688.33 \pm 33.5, \mathrm{P}=0.00008$; invasion: $225.67 \pm 26.10$ vs $400 \pm 43.86, \mathrm{P}=0.004$ ) and MIA-PaCa2 (migration: $63 \pm 5.71$ vs $111 \pm 7.12, \mathrm{P}=0.0017$; invasion: $72.67 \pm 12.50$ vs $173.33 \pm 7.77, \mathrm{P}=0.0002$ ) cells significantly increased after NDRG1 knockdown compared to controls. While the numbers of migrated and invaded PANC-1 (migration: $328.33 \pm 47.06$ vs $152.33 \pm 21.55, \mathrm{P}=0.004$; invasion: $691.3 \pm 66.03$ vs $356 \pm 21.63$, $\mathrm{P}=0.001$ ) and MIA-PaCa2 (migration: $86 \pm 2.94$ vs $44 \pm 9.09$, $\mathrm{P}=0.003$; invasion: $229.7 \pm 23.71$ vs $143.7 \pm 10.21, \mathrm{P}=0.004)$ cells were less than control after NDRG1 overexpression. Taken together, these findings demonstrated NDRG1 prevented pancreatic cancer cell migration and invasion. In CCK-8 proliferation assay, OD450 values were significantly higher than control cells at day 5 after NDRG1 knockdown in PANC-1 and MIA-PaCa2 cell lines. In contrast, the readouts of OD450 declined significantly after NDRG1 overexpression in both cell lines (Fig. 3). These results indicated that NDRG1 had the potential of inhibiting pancreatic cancer cell proliferation, migration and invasion.

Modulation of p-STAT3, MMP2, MMP9, PTEN, $p$-AKT and $P I 3 K$ expression by NDRG1 in pancreatic cancer. Next, we determined the p-STAT3, MMP2, MMP9, PTEN, PI3K and p-AKT after NDRG1 regulation. By western blot assay, p-STAT3, MMP2, MMP9, PTEN, PI3K and p-AKT level were tested after NDRG1 plasmid or shRNA transfection. In both PANC-1 and MIA-PaCa2 cell lines, we observed that
p-STAT3, MMP2, MMP9, PI3K and p-AKT expression were negatively correlated to NDRG1 level while PTEN was positively correlated to NDRG1 overexpression. Specifically, Fig. 4 shows stronger p-STAT3, MMP2, MMP9, PI3K and p-AKT band and much fainter PTEN band in NDRG1 downregulation cell lysates, whereas the opposite were revealed in NDRG1 upregulated pancreatic cancer cells.

\section{Discussion}

Metastasis is the process by which tumor cells move from a primary tumor site to a distal secondary site (15). Plenty of reports have underscored the involvement of oncogenes, tumor suppressor genes and metastasis genes in the occurrence, development and progression of cancer $(16,17)$. NDRG1, a paradoxical gene reported by many studies, plays a metastasis suppressor or a facilitator depending on the type of the tumor.

We found that NDRG1 was involved in proliferation, migration, invasion of pancreatic cancer cell lines in experiments in vitro. Moreover, knockdown of NDRG1 reduced colony formation of pancreatic cancer cells but not significantly. In addition, the effects of NDRG1 on above mentioned cancer behavior might be achieved by activating PTEN and deactivating p-STAT3, MMP2, MMP9, PI3K and p-AKT. Our data indicated that NDRG1 has the propensity to inhibit progression of pancreatic cancer by repressing proliferation and impeding invasion and migration, acting as an antitumor function in pancreatic cancer.

In other pancreas studies on NDRG1, Angst et al found that this protein was highly expressed in well-differentiated cells of pancreatic cancer, whereas the poorly differentiated tumor 
cells were negative. These data suggested that NDRG1 may serve as a marker of differentiation and functioned as a tumor suppressor in the invasive ability and metastasis of cancer cells by inducing differentiation or reversing a metastatic phenotype, which was in agreement with studies of colorectal and prostatic cancer (18). Maruyama et al (19) reported that tumor growth in vivo decreased remarkably by NDRG1 overexpression. Moreover, NDRG1 overproduction could reduce MMP9, a cellular production of angiogenesis-related factors so as to restrict cellular locomotion and invasion ability, those result are similar to our data. They further observed a close association between low NDRG1 expression and poor prognosis in pancreatic cancer patients. MMPs are a family of zinc endopeptidases that cleave ECM molecules and are classified according to their substrate specificity. High levels of MMPs have also been implicated in multiple stages of cancer progression including invasion and migration (20).

STAT3, a central cytoplasmic transcription factor that is activated by phosphorylation in response to extracellular signals and oncogenes, is activated in many human cancers (21), including pancreatic cancer (22). STAT3 activity is positively associated with MMP2 and MMP9 (23), both of which are considered to be downstream of STAT3 pathway (24). They could promote cancer cell migration by degrading the extracellular matrix, and thus regarded as the invasive and metastatic promoter in pancreatic cancer $(25,26)$. In our previous study, we found that activation and blocking of the STAT3 signaling pathway can affect invasion ability and expression of the MMP2 genes in pancreatic cancer $(27,28)$. While in this study, NDRG1 inhibited pancreatic cancer invasion and migration, as well as p-STAT3, MMP2, MMP9 expression. So we assume that the migration and invasion suppressive role of NDRG1 might be attributed to downregulation of p-STAT3, MMP2, MMP9.

PTEN, a phosphatase with opposing function to PI3K, functions as a tumor suppressor to increase PI3K pathway signaling (29) and thus leads to activation of AKT, mTOR signaling pathway (30). The dysregulation of the signaling pathway has been implicated in tumor initiation, cell growth and survival (31). PTEN appears to be a mediator of NDRG1 expression, as silencing of PTEN expression has been shown to downregulate NDRG1 levels, whereas overexpression of PTEN upregulated NDRG1 in a dose- and time-dependent manner (32). However, we found NDRG1 is able to induce PTEN expression. These results indicate that NDRG1 and PTEN may constitute a positive feedloop. This inference was supported by the report that when PI3K signaling overwhelms PTEN signaling, upregulation of NDRG1 restores the coupling of the PI3K and PTEN pathways via upregulation of PTEN and downregulation of PI3K (33). Hence, NDRG1 may synergize with PTEN to decrease pancreatic cancer proliferation through deactivating the PI3K and AKT signaling pathway.

Intriguingly, differing from its tumor suppressive role in pancreatic cancer and some other carcinoma, NDRG1 promotes lung cancer, colon cancer, and liver cancer development. One possible explanation for these conflicting phenomena is that NDRG1 may regulate tumor cell proliferation, migration and invasion through a variety of signaling pathways (34), such as nuclear factor- $\kappa \mathrm{B}(\mathrm{NF}-\kappa \mathrm{B})$, mammalian target of rapamycin (mTOR), and MAPK signaling pathways (35). Future studies in depth are required to identify other signaling pathways that NDRG1 is involved in affecting pancreatic cancer progression.

In conclusion, we have identified NDRG1 as a novel factor that inhibits the growth, invasion and migration of pancreatic cancer. Moreover, NDRG1 appears to play a tumor suppressive role by deactivating STAT3 and PI3K/AKT signaling pathways.

\section{Acknowledgements}

This study was supported by grants from the National Natural Science Foundation of China [no. 81372640 (to Q.Z.J.)].

\section{References}

1. Raimondi S, Maisonneuve P and Lowenfels AB: Epidemiology of pancreatic cancer: An overview. Nat Rev Gastroenterol Hepatol 6: 699-708, 2009.

2. Jemal A, Bray F, Center MM, Ferlay J, Ward E and Forman D: Global cancer statistics. CA Cancer J Clin 61: 69-90, 2011.

3. Han H and Von Hoff DD: SnapShot: Pancreatic cancer. Cancer Cell 23: 424-424 e421, 2013.

4. Nieto J, Grossbard ML and Kozuch P: Metastatic pancreatic cancer 2008: Is the glass less empty? Oncologist 13: 562-576, 2008.

5. Song Y, Lv L, Du J, Yue L and Cao L: Correlation of N-myc downstream-regulated gene 1 subcellular localization and lymph node metastases of colorectal neoplasms. Biochem Biophys Res Commun 439: 241-246, 2013.

6. Chang X, Xu X, Ma J, Xue X, Li Z, Deng P, Zhang S, Zhi Y, Chen J and Dai D: NDRG1 expression is related to the progression and prognosis of gastric cancer patients through modulating proliferation, invasion and cell cycle of gastric cancer cells. Mol Biol Rep 41: 6215-6223, 2014.

7. Wang J, Cai J, Li Z, Hu S, Yu L, Xiao L and Wang Z: Expression and biological function of $\mathrm{N}$-myc down-regulated gene 1 in human cervical cancer. J Huazhong Univ Sci Technolog Med Sci 30: 771-776, 2010.

8. Wang B, Li J, Ye Z, Li Z and Wu X: N-myc downstream regulated gene 1 acts as a tumor suppressor in ovarian cancer. Oncol Rep 31: 2279-2285, 2014.

9. Jin R, Liu W, Menezes S, Yue F, Zheng M, Kovacevic Z and Richardson DR: The metastasis suppressor NDRG1 modulates the phosphorylation and nuclear translocation of $\beta$-catenin through mechanisms involving FRAT1 and PAK4. J Cell Sci 127: 3116-3130, 2014.

10. Chen Z, Zhang D, Yue F, Zheng M, Kovacevic Z and Richardson DR: The iron chelators Dp44mT and DFO inhibit TGF- $\beta$-induced epithelial-mesenchymal transition via up-regulation of N-Myc downstream-regulated gene 1 (NDRG1). J Biol Chem 287: 17016-17028, 2012.

11. Liu W, Iiizumi-Gairani M, Okuda H, Kobayashi A, Watabe M, Pai SK, Pandey PR, Xing F, Fukuda K, Modur V, et al: KAI1 gene is engaged in NDRG1 gene-mediated metastasis suppression through the ATF3-NFkappaB complex in human prostate cancer. J Biol Chem 286: 18949-18959, 2011.

12. Guan RJ, Ford HL, Fu Y, Li Y, Shaw LM and Pardee AB: Drg-1 as a differentiation-related, putative metastatic suppressor gene in human colon cancer. Cancer Res 60: 749-755, 2000

13. Lu WJ, Chua MS, Wei W and So SK: NDRG1 promotes growth of hepatocellular carcinoma cells by directly interacting with GSK- $3 \beta$ and Nur77 to prevent $\beta$-catenin degradation. Oncotarget 6: 29847-29859, 2015.

14. Wang Q, Li LH, Gao GD, Wang G, Qu L, Li JG and Wang CM: HIF-1 $\alpha$ up-regulates NDRG1 expression through binding to NDRG1 promoter, leading to proliferation of lung cancer A549 cells. Mol Biol Rep 40: 3723-3729, 2013.

15. Stafford LJ, Vaidya KS and Welch DR: Metastasis suppressors genes in cancer. Int J Biochem Cell Biol 40: 874-891, 2008.

16. Valastyan S and Weinberg RA: Tumor metastasis: Molecular insights and evolving paradigms. Cell 147: 275-292, 2011.

17. Yokota J: Tumor progression and metastasis. Carcinogenesis 21: 497-503, 2000. 
18. Angst E, Sibold S, Tiffon C, Weimann R, Gloor B, Candinas D and Stroka D: Cellular differentiation determines the expression of the hypoxia-inducible protein NDRG1 in pancreatic cancer. $\mathrm{Br}$ J Cancer 95: 307-313, 2006.

19. Maruyama Y, Ono M, Kawahara A, Yokoyama T, Basaki Y, Kage M, Aoyagi S, Kinoshita $\mathrm{H}$ and Kuwano M: Tumor growth suppression in pancreatic cancer by a putative metastasis suppressor gene Cap43/NDRG1/Drg-1 through modulation of angiogenesis. Cancer Res 66: 6233-6242, 2006.

20. Egeblad M and Werb Z: New functions for the matrix metalloproteinases in cancer progression. Nat Rev Cancer 2: 161-174, 2002.

21. Wang L, Luo J and He S: Induction of MMP-9 release from human dermal fibroblasts by thrombin: Involvement of JAK/ STAT3 signaling pathway in MMP-9 release. BMC Cell Biol 8: 14, 2007.

22. Li H, Huang C, Huang K, Wu W, Jiang T, Cao J, Feng Z and Qiu Z: STAT3 knockdown reduces pancreatic cancer cell invasiveness and matrix metalloproteinase-7 expression in nude mice. PLoS One 6: e25941, 2011.

23. Huang X, Dai S, Dai J, Xiao Y, Bai Y, Chen B and Zhou M: Luteolin decreases invasiveness, deactivates STAT3 signaling, and reverses interleukin-6 induced epithelial-mesenchymal transition and matrix metalloproteinase secretion of pancreatic cancer cells. Onco Targets Ther 8: 2989-3001, 2015.

24. Xuan X, Li S, Lou X, Zheng X, Li Y, Wang F, Gao Y, Zhang H, $\mathrm{He} \mathrm{H}$ and Zeng Q: Stat3 promotes invasion of esophageal squamous cell carcinoma through up-regulation of MMP2. Mol Biol Rep 42: 907-915, 2015.

25. Qian LW, Mizumoto K, Urashima T, Nagai E, Maehara N, Sato N, Nakajima M and Tanaka M: Radiation-induced increase in invasive potential of human pancreatic cancer cells and its blockade by a matrix metalloproteinase inhibitor, CGS27023. Clin Cancer Res 8: 1223-1227, 2002.

26. Fukuda A, Wang SC, Morris JP IV, Folias AE, Liou A, Kim GE Akira S, Boucher KM, Firpo MA, Mulvihill SJ, et al: Stat3 and MMP7 contribute to pancreatic ductal adenocarcinoma initiation and progression. Cancer Cell 19: 441-455, 2011.
27. Huang C, Jiang T, Zhu L, Liu J, Cao J, Huang KJ and Qiu ZJ: STAT3-targeting RNA interference inhibits pancreatic cancer angiogenesis in vitro and in vivo. Int J Oncol 38: 1637-1644, 2011.

28. Huang C, Yang G, Jiang T, Huang K, Cao J and Qiu Z: Effects of IL-6 and AG490 on regulation of Stat 3 signaling pathway and invasion of human pancreatic cancer cells in vitro. J Exp Clin Cancer Res 29: 51, 2010.

29. Zhang S and Yu D: PI(3)king apart PTEN's role in cancer. Clin Cancer Res 16: 4325-4330, 2010

30. Mukherjee A, Samanta S and Karmakar P: Inactivation of PTEN is responsible for the survival of Hep G2 cells in response to etoposide-induced damage. Mutat Res 715: 42-51, 2011.

31. Wallin JJ, Edgar KA, Guan J, Berry M, Prior WW, Lee L, Lesnick JD, Lewis C, Nonomiya J, Pang J, et al: GDC-0980 is a novel class I PI3K/mTOR kinase inhibitor with robust activity in cancer models driven by the PI3K pathway. Mol Cancer Ther 10: 2426-2436, 2011

32. Bandyopadhyay S, Pai SK, Hirota S, Hosobe S, Tsukada T, Miura K, Takano Y, Saito K, Commes T, Piquemal D, et al: PTEN up-regulates the tumor metastasis suppressor gene Drg-1 in prostate and breast cancer. Cancer Res 64: 7655-7660, 2004.

33. Kovacevic Z, Chikhani S, Lui GY, Sivagurunathan S and Richardson DR: The iron-regulated metastasis suppressor NDRG1 targets NEDD4L, PTEN, and SMAD4 and inhibits the PI3K and Ras signaling pathways. Antioxid Redox Signal 18: 874-887, 2013.

34. Hu ZY, Xie WB, Yang F, Xiao LW, Wang XY, Chen SY and Li ZG: NDRG1 attenuates epithelial-mesenchymal transition of nasopharyngeal cancer cells via blocking Smad2 signaling. Biochim Biophys Acta 1852: 1876-1886, 2015.

35. Sun J, Zhang D, Bae DH, Sahni S, Jansson P, Zheng Y, Zhao Q, Yue F, Zheng M, Kovacevic Z, et al: Metastasis suppressor, NDRG1, mediates its activity through signaling pathways and molecular motors. Carcinogenesis 34: 1943-1954, 2013. 